\title{
A Survey: A Social Explanation System Applied to Group Recommendations
}

\author{
Aakanksha Thakur \\ M.Tech. Research Scholar, Department of CSE \\ SIRTS, Bhopal
}

\author{
Chetan Gupta \\ Assistant Prof., Department of CSE \\ SIRTS, Bhopal
}

\begin{abstract}
Recommendation systems are currently successful solutions to enable online users to access information that meets their preferences and needs in congested environments. In recent years, various methods have been developed to improve their performance. This paper provide an overview of the use of fuzzy tools in recommended systems to identify common research topics and the pillars examined and to identify candidates for future research lines to support current societal developments. Based recommendation systems there is a need to research analytical analytics systems that design the design and development of the reporting system, not just the latest products. This design and development process uses analysis, visual design analysis, information modification approaches, and scientific research. In addition, experiments are required to determine the impact of these systems on learning behaviour, its range, and capabilities to add to the small evidence available.
\end{abstract}

\section{Keywords}

Recommender systems, visualization, user preferences, fuzzy logic.

\section{INTRODUCTION}

Recommender systems can be defined as programs that attempt to provide specific users (people or companies) with the most appropriate elements (products or services) by asking about a user's interest in an element based on the information about the elements, Users and the interactions between them speak articles and users. The goal of development systems is to read information by retrieving the most relevant information and services from many appointments, thereby providing personalized services. The key feature of a recommended system is its ability to generate user preferences and interests by analysing its behaviour and the behaviour of other users using personalized recommendations [1]. Eservice personnel techniques are characterized by recommender systems that have attracted much attention over the past 20 years. Early recommender system research relied on information and research filtering. The independent recommendation system developed in the mid-nineties developed as an independent research area, as the researchers focused explicitly on referral issues due to the rating structure. General technical techniques include collaborative filtering (CF), content-based (CB) and knowledge-based (KB) techniques. Each tendering approach has advantages and limitations. For example, CF has specific scalability and cold start issues, while CB recommends recommendations. Numerous presentations have been made to address these issues, such as non-social recommendation systems, fuzzy attorney systems, contextual recommendation systems, and group referencing systems.

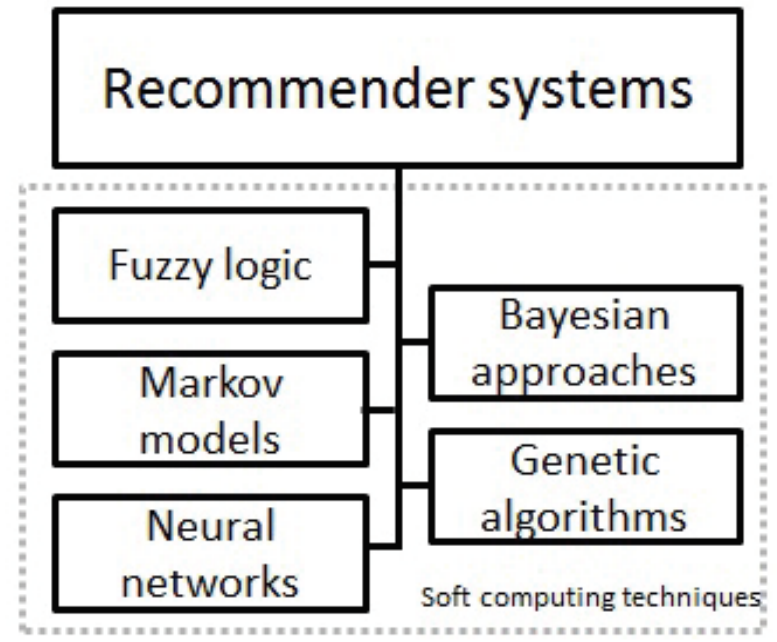

Fig. 1: Soft Computing Approaches in Recommender Systems Development

One of the most commonly used paradigms for performing personalization processes to provide users with the information tools that preserve and impose their needs in an overburdened digital world is advertising systems. Although primarily named after ecommerce areas, today they have been extended to include various scenarios such as e-learning, tourism, libraries, e-government, financial investment, and other applications. According to the available information used to provide generation suggestions, recommender systems may be divided into various recommendation paradigms. Originally, demographic terms are the most important, as such information is available. Recently, however, the two main paradigmatic addresses are the recommendation for collaborative filters [8] and the content Recommendation

a. Collaborative Filtering Systems: Can only use recommendations from user administrators and without additional information. In its most basic approach, collaborative filtering focuses on the proposal to the target counter, the elements that are already being used by other users with the same preferences.

b. Content Based Recommendation: The innovation company focuses on using additional information outside of the user managers (eg article attributes) to characterize articles, thus displaying articles with identical characteristics to those that the user has requested in the past [8]. 


\subsection{Recommender Systems and Social Networks}

The techniques most commonly used in command systems are content-based (CB) filtering and collaborative filtering (CF). Each user in such systems is typically represented by a user profile consisting of items that that user rated (or purchased). Content-based filtering provides new points based on their similarity to elements already in profile. To achieve this, more details are required for each article. On the other hand, collaborative filtering approaches to the elements are agnostic. Instead, they use the user's ratings to find other similar users (or items) based on their word patterns. The general nature of collaborative filtration systems has been the reason for their great success, as they have been used to deliver a wide range of products such as movies, music, news, books, research articles, search queries, social tags, and more. Although a bipartite user object that uses ratings as the sole input for such systems is no longer sufficient, a solid structure is no longer sufficient to expose all available information such as content, context, social information, and metadata. As a result, many problems have been created to include more information about the user, his context (spatial, temporal, social, etc.) and its evolution over time [12].

\subsubsection{Beyond user-item ratings:}

Context-aware recommender systems (CARS) generate more relevant alerts by adapting to the specific context of the user. The contextual factors that a recommender system must address relate to the time, place and purpose of the targeting user. The user context may be according to statistics or may change over time. CARS receives a pre filter tip, which uses context information to select relevant elements, a classic recommendation that is relevant according to the previous reviews, and a re-contextual filtering step that filters and outputs the output. The traditional commander

\subsubsection{Beyond simple item recommendations}

Recommended systems (RS) have become popular because they can personalize the user experience based on automated recommendations. They appeared for the first time in the ebusiness, with which particularly interested persons were led to buy products, which are of interest for the customers. However, in recent years, other areas such as digital news collections, such as news and research articles, database queries or even web services, have become more widely used.

\subsubsection{Beyond simple evaluation metrics}

The development of an effective stimulated system is not just limited to providing preferences to users and recommending the most eye-catching items for each user. Especially in the ecommerce system, where new items are added in a quick tool, it is important that the recommender systems switch popularly to newly imported items with similar functions, but only the few available levels. This challenge is defined in the literature as a long-tail problem and can use content-based methods to determine the similarity between popular and newer articles and new articles, or to replace popular articles, or to replace the article in a long-tailed way. Tail clusters summarize scant reviews with aggregated reviews per cluster. In general, the same companion that solves the cold start problem will be used to try out the long term problem.

\section{LITERATURE REVIEW}

Jie Lu, Dianshuang Wu, MingsongMao,Wei Wang, Guangquan Zhang [1], A recommender system aims to make users with personal online products or services overburden the increase in online problems and improve the management of customer images. Various display system technologies have been postponed since the mid-nineties and, lately, many types of recommender system software have been developed for a variety of applications. Researchers and managers recognize that referral systems offer great opportunities and problems for businesses, government agencies, educational institutions and other areas, with recent successes in seeing recommendations from practitioners. It is so important that a high-quality, instructive review of current trends be given not only to theoretical research but, more importantly, to practical developments in recommender systems.

This paper needs to be compared again with the existing application system for recommendation systems, whose applications are released in eight main categories: egovernment, e-business, e-commerce / e-commerce, e-library, e-commerce, e-tourism, e -group resource services and activities and summarizes the suggested techniques used in each category. They hope that this document will provide researchers and practitioners with up-to-date knowledge on the recommendation of program requests and guidelines for the development and application of design and application systems in various fields to assist users in various decisionmaking processes. Two important features of this paper distinguish it from other research papers in the field of system-accompanying systems: 1) It focuses on the realistic attack development of recommendation systems; 2) systematically researching reporting capabilities (online software) into four dimensions: recommendation methods (such as $\mathrm{CF}$ ).

Chen He, Denis Parra, KatrienVerbert [5] Recommender systems have been extensively studied in recent decades. As different algorithms are developed and used in different domain applications, recent research schemes are increasingly focusing on the user experience of recommender systems. This research focuses on recommendation algorithms and focuses on several human factors that influence the acceptance of recommendations, such as: Eg user satisfaction, trust, transparency and sense of command. In this paper, they create an interactive visual alignment scheme that combines recommendations with visualization techniques to support people-to-people engagement and referrals.

Then analyse existing interactive recommendation files according to the dimensions of hit. Although many interesting systems have been developed in this field, many challenges still need to be addressed. First, research objectives such as the loss of cold start, diversity, news, and serendipity of recommendations are still under investigation. Second, there is a need to adjust the level of control and build the visualization technology used for different user alarms, as innovative visualizations are too complete for a broad audience. Thirdly, the different techniques have to be compared and counteracted with a common evaluation framework, and design guidelines have to be developed. The use of visual visualizations that they analysed in this article provides an interesting starting point with many ideas, but developed draft guidelines that examine how technologies are suitable for different users and institutions to help new researchers and practices. 
Lara Quijano-Sanchez, Christian Sauer b, JuanA. RecioGarcia, Belen Diaz-Agudo [7] Recommendation systems, which help users determine which elements to choose from in a variety of choices, are mainly due to their needs and preferences. In this context, the explanations refer to supplemental information that can help participants better understand the performance of the system and promote goals such as trust, confidence in decision-making or benefits. In this post they protest against a personal social individual declaration (PSIE). Unlike other business systems, the PSIE proposal includes a brief storyline for grouping groups and an explanation of the social reality of the group in order to achieve a positive response to a better reception of the group. Brings the group recommendation. For other issues, they develop a special need to learn directly about tactful statements as the personal relationships of the users in a group are adjusted and personalized perfume declarations are encouraged, which encourage the users to accept the recommended recommendations.

In this article, they have a Personal Social Customization (PSIE), in which they suggest to users of the group months why the system assumes that the recommended item is the best option for the group. The presented study introduces a romantic method of limiting group dynamics to other works that limit the explanation of a group tempo to the insignificant process of justice of the selected aggregation technique. This new perspective relates the exploration study to the social behaviour of users in the group. Previously, social declarations were taken for single pointers, but to the best of the authors' knowledge, never for group recipients. The proposed approach focuses in particular on explaining the recommendation process resulting from the Happy Movie system.

RacielYera 1, Luis Mart'inez [8] Recommender systems are currently successful solutions to enable online users to access information that meets their preferences and needs in congested environments. In recent years, various methods have been developed to improve their performance. This article aims to provide an overview of the use of fuzzy tools in recommended systems to identify common research topics and the pillars examined and to identify candidates for future research lines to support current societal developments based recommendation systems. This study analysed more than one hundred articles aimed at using recommendation techniques for the fuzzy support system. In the past, this work was organized into three large groups according to three different recommendation paradigms (content-based, optional-based collaboration filtering and model-based collaboration filtering), including several subgroups of the core applications used in the respective works. Finally, future research occupations are considered in fuzzy referral systems, which focus on developing a common framework, developing assessment scenarios based on fuzzy information business recommendations, and developing new emerging sources of information (eg, networks) and solving new problems in recommender systems. Surveys (eg. grouping, natural noise management).
Xiaopeng Li, James She [11] Modern systems use normal collaborative filtering with rating information to indicate requirements to users based on their successful performance. However, due to the bad inconvenience of collaboration, such as sparsity, cold start, etc., a. Reference is made to hybrid methods that take into account both rating and content information. Most of the previous work in this area cannot be learned for a good presentation of the content for the referral task, or just for the textual content. Therefore, their methods are very limited in the current multimedia scenario. In the work of is, a Cooperative Variable Automatic Encoder (CVAE) is proposed for a generative model of the Bayesian model that considers the evaluation and content for the recommendation in a multidisciplinary scenario.

The model learns deeply latent representations of content data in an opaque manner and also teaches implicit relationships between elements and users of both content and rating. .is paper sets up the automatic encoder for the collaborative variant, which generates content in conjunction with the implementation of implicit relationships between elements and users. It is a probabilistic Bayesian generation model that combines collaborative and content-related information through randomly-based learning models and graphical models to achieve robust presentation preferences. Due to its Bayesian nature, effective variant influences are derived from the stochastic diversity of the Bayesian variants.

Magdalini Eirinaki, Jerry Gao, IraklisVarlamis, Konstantinos Tserpes [12] Social networks have become very important for networking, communication and layout. Social networking applications generate a lot of data on a daily basis, and social networks are becoming a growing field of research because of their data heterogeneity and structure in shape, size, and dynamics. If this data domain is provided by recommender systems, the result of linking links may raise interesting issues related to social gathering, membership, and referrals from friends. In this paper they look at the different facets of big social recommendation systems, summarizing the challenges and interesting questions and discussions of the solutions. A large body of research for collaborative filters beyond matrix delay is based on non-negative matrix factorization (NMF) and tensor decomposition of the evaluation matrix.

It also proposed a great and graphical NMF regularization to encapsulate information from the social diagram into the evaluation model and to reduce and eliminate the special requirements for large graphics. The Laplace diagram of regulatory requirements has been replaced by other regulations. With the growing list of possible recommendations, the validation of algorithms as well as spatial indications increases over time. Besides the justification, variety, coincidence, novelty and timeliness of the recommended articles and user skills as well as the removal of user readings are some of the new criteria for the evaluation of the quality of the recommendation. 
Table 1: Analysis of the Available Recent Algorithms

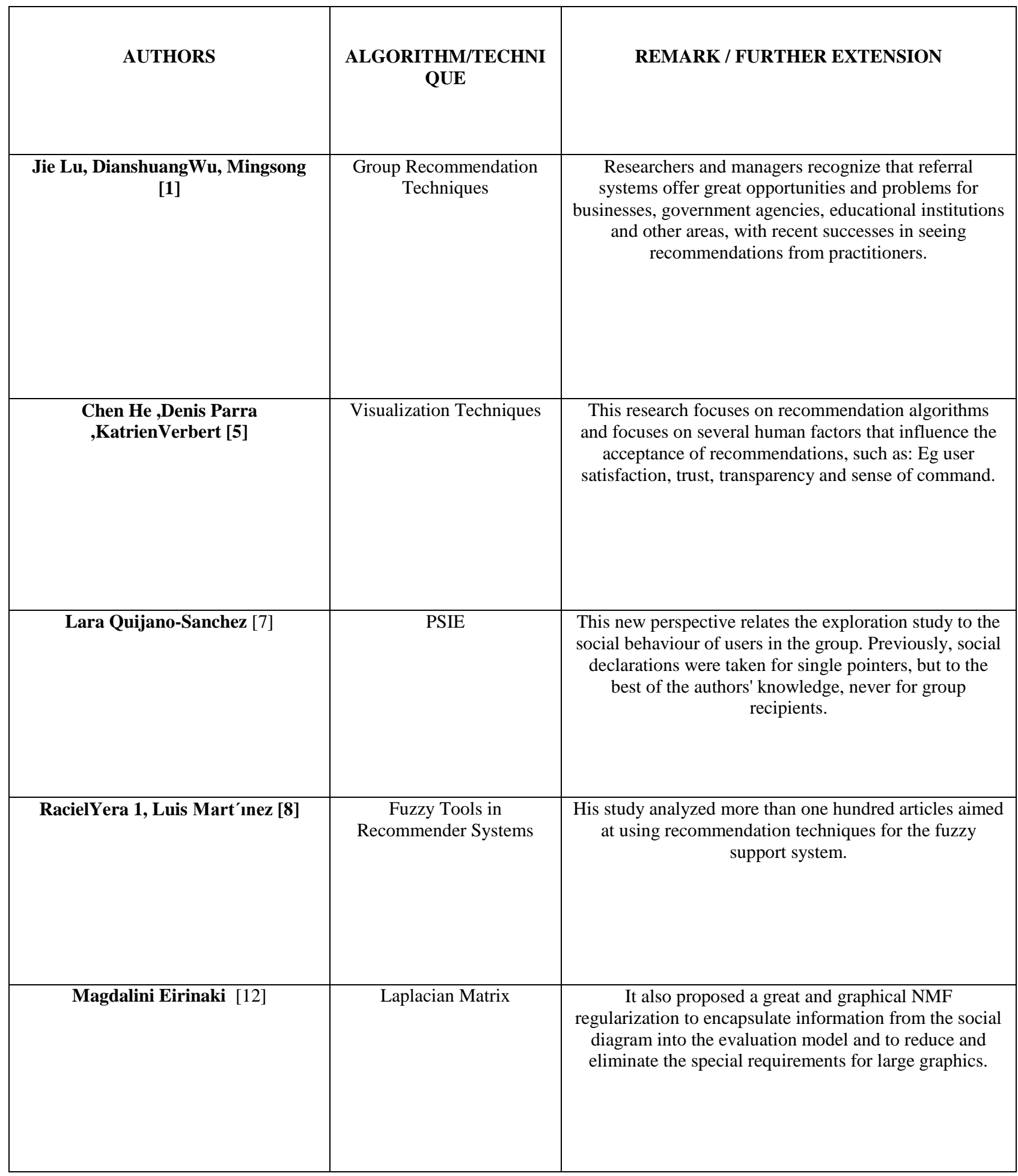


In the comparison Table 1 above, some existing recent algorithms are discussed, their techniques and remarks.

\section{PROPOSED APPROACH}

\section{Stochastic Function:}

This is the random function manner formulae of processing data, this works to process the randomly selected element from the available data community dataset. A stochastic process can be denoted, among other ways, by $\mathrm{X}(\mathrm{t})$ belongs to $\mathrm{X}$, where $\mathrm{t}$ is the randomly selected element. \{ldisplaystyle $\left.\backslash\{\mathrm{X}(\mathrm{t}) \backslash\}_{-}\{\mathrm{t} \operatorname{lin} \mathrm{T}\}\right\}$

\section{DDQP (Distance dynamic QLearning Partitioning)}

Approach:

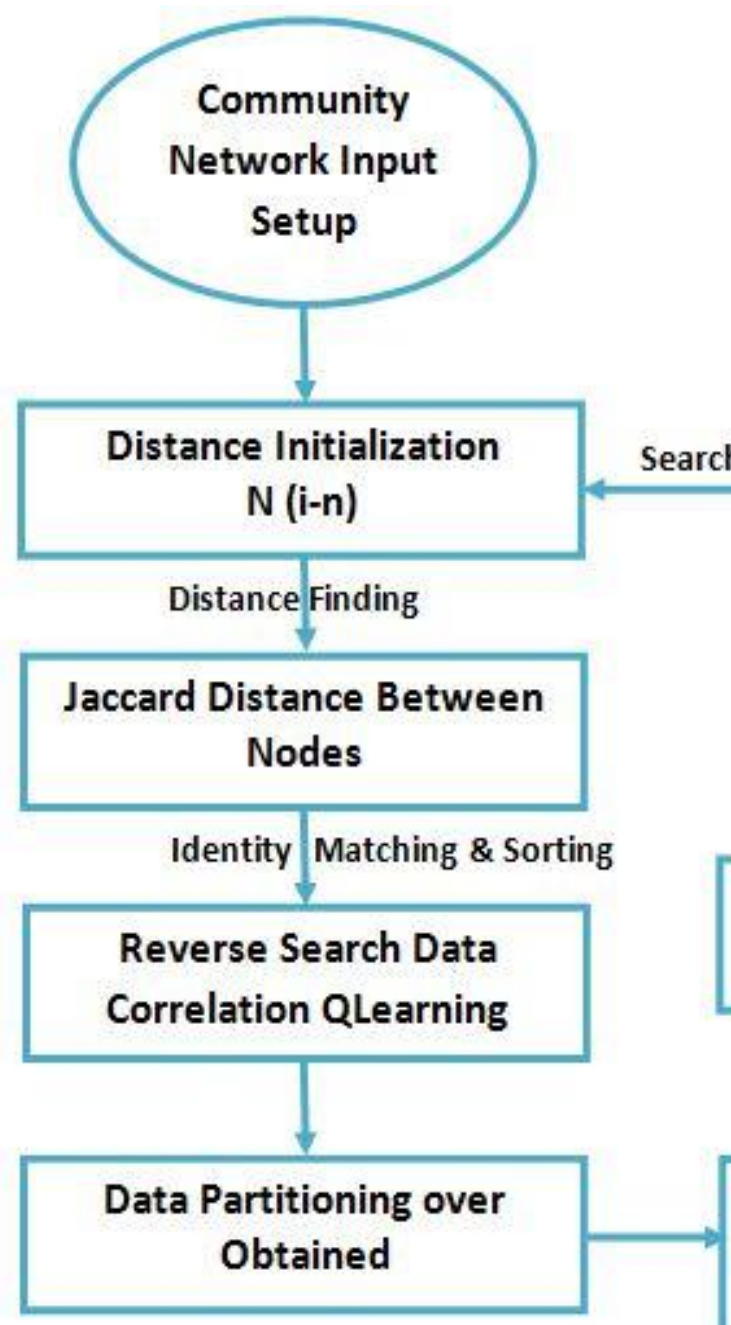

Phase 1: Community traversing management- Identity management specify to the development of employing arriving technologies to manage information concerning the identity of people, monitoring reverse phase information. The aim of identity management is to process the data in forward and backward manner. Further finding their relation and making them partition to sub community.

Phase 2: Data Analysis- In second phase generated data will be used to find sub community based on their node weight. The node weight value help in sub partitioning and getting sub community over the detected community from phase 1 above.

Phase 3: Arranging them according to QLearning- At this end QLearning help in arranging according to its privilege.

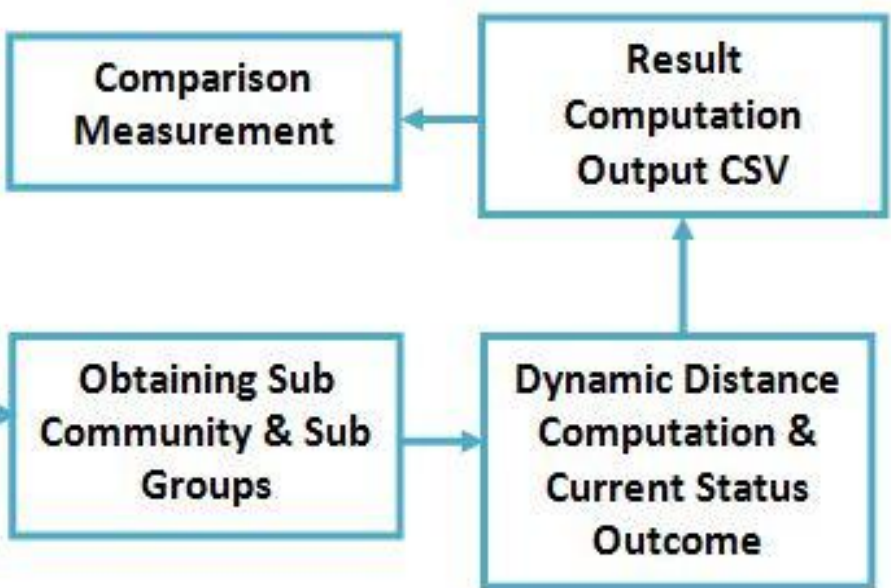

Fig.2: Flow Chart of Overall Execution Process

Steps Taken for the work performance:

1. An extraction of network input data and processing it for the further community searching detection algorithm by extending Attractor approach.

2. By working towards distance dynamic and finding node network distance using Jaccard distance algorithm.

3. Matching the identity, community sorting and further applying QLearning for reverse detection over obtained distance dynamic data.
4. Generating cost using the cost function and matching it, if it is eligible for the partitioning and cost function computed value is less than partitioning value factor $\mathrm{P}$, then it is allowed to partitioning in the platform provided.

5. Further an analysis over the node and community user of different group is performed. 


\section{CONCLUSION}

There are still many challenges that need to be addressed. First, research objectives such as the loss of cold start, diversity, news, and serendipity of recommendations are still under investigation. Second, there is a need to adjust the level of control and create the visualization technology used for different user markings, e.g. For example, innovative visualizations may be too complex for a broad audience. Thirdly, the different techniques have to be compared and counteracted with a common evaluation framework, and design guidelines have to be developed. The use of visual visualizations that analyzed in this article provides an interesting starting point with many ideas, but developed draft guidelines that examine how technologies are suitable for different users and institutions to help new researchers and practices and hope that these ideas will help to improve and remove research into interactive registration opportunities.

\section{REFERENCES}

[1] Jie Lu , DianshuangWu, MingsongMao,Wei Wang, Guangquan Zhang, "Recommender system Application Developments: A Survey", Decision Systems \& EService Intelligence Lab, Centre for Quantum Computation \& Intelligent Systems, Faculty of Engineering and Information Technology, University of Technology Sydney, Australia 2015.

[2] Jorge Castro, Francisco J. Quesada, 'an Palomares, LuisMart'ınez, "A Consensus-Driven Group Recommender System" International Journal of Intelligent Systems, Vol. 00, 1-20 (2015) 2015 Wiley Periodicals.

[3] Nick Hajli, "Social Commerce Constructs and Consumer's Intention to Buy Newcastle" International Journal of Information Management, University Business School, United Kingdom 2015.

[4] Nguyen Tho Thong, Le Hoang Son, "HIFCF: An Effective Hybrid Model Between Picture Fuzzy Clustering and Intuitionist Fuzzy Recommender Systems for Medical Diagnosis" VNU University of Science, Vietnam National University, Hanoi, Viet Nam, Elsevier 2015.

[5] Chen He ,Denis Parra ,Katrien Verbert, "Interactive Recommender Systems: A Survey of the State of the Art and Future Research Challenges and Opportunities" Expert Systems with Applications , Elsevier 2016.

[6] Huong May Truong, "Integrating Learning Styles and Adaptive E-learning System: Current Developments, Problems and Opportunities" Corvinno Technology Transfer Centre, Eduworks ITN, Kozraktar12/a, H1093Budapest, Hungary, Elsevier 2016.

[7] Lara Quijano-Sanchez, Christian Sauer, Juan A. RecioGarcia, Belen Diaz-Agudo, "Make it personal: A social explanation system applied to group recommendations Big Data", Universidad Carlos III de Madrid, Getafe, Spain, School of Computing and Engineering, University of West London, London, United Kingdom Facultad de Informatica, Universidad Complutense de Madrid, Madrid, Spain, Elsevier 2017.

[8] Raciel Yera, Luis Martinez, "Fuzzy Tools in Recommender Systems: A Survey" International Journal of Computational Intelligence Systems, Vol. 10, 776-80, 2017.

[9] Robert Bodily and Katrien Verbert, "Review of Research on Student-Facing Learning Analytics Dashboards and Educational Recommender Systems", IEEE Transactions on Learning Technologies, TLT-2017-03-0046, IEEE August 2017

[10] Stefan Feyer, Sophie Siebert, BelaGipp, Akiko Aizawa, and Joeran Beel, "Integration of the Scientific Recommender System" Mr. DLib into the Reference Manager JabRef, European Conference on Information Retrieval, University of Konstanz, Konstanz, Germany, Springer 2017.

[11] Xiaopeng Li, "Collaborative Variation Autoencoder for Recommender Systems" KDD'17, KDD '17 Proceedings of the 23rd ACM SIGKDD International Conference on Knowledge Discovery and Data Mining, Halifax, NS, Canada, August 13-17, 2017.

[12] Magdalini Eirinak, Jerry Gao, Iraklis Varlamis, Konstantinos Tserpes, "Recommender Systems for Large-Scale Social Networks: A Review of Challenges and Solutions" A Computer Engineering Department, San Jose State University, San Jose, CA, USA JAN 2018.

[13] M. Kosinski, D. Stillwell, and T. Graepel. "Private Traits and Attributes are Predictable from Digital Records of Human Behavior "In Proceedings of the National Academy of Sciences, 2013.

[14] H. Ma, D. Zhou, C. Liu, M. R. Lyu, and I. King. "Recommender Systems with Social Regularization". In Proc. WSDM '11 Proceedings of the fourth ACM International Conference on Web Search and Data Mining, Pages 287-296, 2011.

[15] P. Pu and L. Chen. "Trust Building with Explanation Interfaces". In Proc. IUI '06 Proceedings of the 11th international conference on Intelligent user interfaces, pages 93-100, 2006.

[16] M. Rodriguez, C. Posse, and E. Zhang. "Multiple Objective Optimizations in Recommender Systems". In Proc RecSys ' 12 Proceedings of the sixth ACM Conference on Recommender Systems, pages 11-18, 2012.

[17] Amit Sharma and Dan Cosley. "Network-Centric Recommendation: Personalization with and in Social Networks". In International Conference on Privacy, Security, Risk, and Trust, and IEEE International Conference on Social Computing pages 282-289, IEEE 2011. 